\title{
Three-dimensional phosphorus-doped graphene as an efficient metal-free electrocatalyst for electrochemical sensing
}

Ye Tian ${ }^{1 *}$, Zhen Wei ${ }^{2}$, Kehui Zhang ${ }^{3}$, Shuo Peng ${ }^{4}$, Xiao Zhang ${ }^{4}$, Wuming Liu ${ }^{5}$, $\mathrm{Ke} \mathrm{Chu}{ }^{6 *}$

${ }^{1}$ Department of Physics, College of Science, Hebei North University, Zhangjiakou 075000, Hebei, China

${ }^{2}$ Department of Chemistry, College of Science, Hebei North University, Zhangjiakou 075000, Hebei, China

${ }^{3}$ College of Electrical Engineering, Hebei University of Architecture and Engineering, Zhangjiakou 075000, Hebei, China

${ }^{4}$ College of Information Science and Engineering, Hebei North University, Zhangjiakou 075000, Hebei, China

${ }^{5}$ Institute of Physics, Chinese Academy of Sciences, Beijing 100190, China

${ }^{6}$ Department of Materials Science and Engineering, School of Mechatronic Engineering, Lanzhou Jiaotong University, Lanzhou 730070, Gansu, China

*Corresponding author. E-mail address: tianyehnu@163.com (Y.Tian); chukelut@163.com (K. Chu) 


\section{Abstract}

Three-dimensional (3D) porous architecture enables the high exploration of graphene excellent properties, i.e. large surface area and superior conductivity. Additionally, heteroatoms doping can improve the electrocatalytic activity of graphene by creating abundant defective/active sites. To combine the prominent advantages of $3 \mathrm{D}$ porous structure and heteroatoms doping, we reported the facile synthesis of 3D phosphorus (P)-doped graphene (3D-PG) and evaluated its potential application in electrochemical $\mathrm{H}_{2} \mathrm{O}_{2}$ sensing. As an efficient metal-free electrocatalyst, 3D-PG modified electrode exhibited exceptional sensing performances for sensitive, selective and stable detection of $\mathrm{H}_{2} \mathrm{O}_{2}$, which was ascribed to the synergistic effects of 3D porous structure and P-doping in significantly enhancing the eletrocatalytic activity for $\mathrm{H}_{2} \mathrm{O}_{2}$ reduction reaction. More importantly, the 3D-PG based sensor could be used for real-time tracking $\mathrm{H}_{2} \mathrm{O}_{2}$ released from living HeLa cells, hence making 3D-PG a potential high-performance electrocatalyst for practical electrochemical sensing.

Keywords: Three-dimensional graphene; Phosphorus-doped graphene; Electrocatalytic activity; Electrochemical sensor 


\section{Introduction}

Electrochemical sensors have been widely applied in the fields of clinical diagnosis, environmental protection, pathological research and food industry owing to their low cost, fast response, high sensitivity, and convenience in operation[1-3]. In the construction of electrochemical sensors, the electrocatalytic ability of electrocatalysts is crucial in determining the performance of electrochemical sensors[4]. Noble metals [5] and transition-metal oxides[6] have long been considered as the most efficient electrocatalysts for electrochemical sensing due to their excellent catalytic activities. However, high price and poor stability of noble metals, and low electric conductivity of transition-metal oxides largely limit their practical applications[4]. Therefore, there is considerable interest to develop efficient metal-free electrocatalysts with large surface area, efficient charge transfer and remarkably electrocatalytic activities.

Recently, graphene-based materials have been extensively investigated as efficient electrocatalysts to develop electrochemical sensors and biosensors due to their unique two-dimensional (2D) structures, large specific surface area, excellent electrical conductivity, good biocompatibility and ease of fictionalizations and production[4, 7]. It has been reported that graphene-based sensors exhibit high sensitivity for the detection of different analyses of $\mathrm{H}_{2} \mathrm{O}_{2}$ [8], glucose[9], dopamine[10], paracetamol[11], trinitrotoluene[12], phenol[13], etc. However, the tendency of graphene aggregations due to strong $\pi-\pi$ interlayer interactions leads to the significant degradation of graphene outstanding properties. To overcome this 
limitation, an efficient way is to assemble 2D graphene sheets into three-dimensional (3D) architectures (i.e., hydrogels, aerogels, sponges, and foams)[14-16]. By virtue of its 3D porous structure with interconnected networks, 3D graphene possesses extremely high surface areas, robust mechanical flexibility, and rapid charge/mass transfer kinetics as compared to 2D graphene. Hence, 3D graphene holds great promise for developing highly sensitive electrochemical sensors[17].

On the other hand, recent progress has demonstrated that the doping of heteroatoms $(\mathrm{N}, \mathrm{P}, \mathrm{B})$ into graphene lattice can cause the redistribution of spin and charge densities and further enhance the electrocatalytic properties of graphene[18-20]. It has been reported that abundant defective/active sites created by doped heteroatoms dramatically contribute to the sensing performances of modified electrodes[20]. Therefore, it is anticipated that the combination of 3D graphene structure and heteroatoms doping is beneficial to greatly improve the electrochemical sensing performances. For instance, the electrochemical sensors based on 3D graphene with $\mathrm{N}$-doping have been successfully applied for the detection of $\mathrm{H}_{2} \mathrm{O}_{2}$ [21], glucose[22] and dopamine[23], exhibiting excellent sensitivity and selectivity.

$\mathrm{N}$-doped graphene (NG) has been widely studied among the heteroatoms doped graphene materials[24]. P-doped graphene (PG) has attracted growing interest in recent three years [25-28]. It has been revealed that PG could exhibit a comparable or even superior electrocatalytic activity to NG because of its stronger electron-donating ability[25]. As such, PG offers great opportunities for potential applications in fuel cells[25], supercapacitors[29], and lithium-ion batteries[25, 30]. Nevertheless, PG has 
rarely been studied for the construction of electrochemical sensors. To the best of our knowledge, there is no report on the preparation of 3D structural PG (3D-PG) for electrochemical sensing.

In this work, we reported the first synthesis of 3D-PG through a self-assembly hydrothermal method and subsequent annealing treatment. Self-assembly hydrothermal method is believed as one of the most attractive strategies in building up 3D porous structure because of its simplicity, low cost, mild experimental conditions and scale-up synthesis[31]. As a proof of concept, the as-prepared 3D-PG was then used as an efficient metal-free electrocatalyst for $\mathrm{H}_{2} \mathrm{O}_{2}$ sensing. Benefiting from the synergistic effects of 3D porous framework and P-doping, it was demonstrated that 3D-PG modified electrode exhibited much improved catalytic activity for $\mathrm{H}_{2} \mathrm{O}_{2}$ reduction reaction and good sensing performances for highly sensitive detection of $\mathrm{H}_{2} \mathrm{O}_{2}$. Furthermore, 3D-PG was successfully applied to determine the trace-level $\mathrm{H}_{2} \mathrm{O}_{2}$ released from living HeLa cells.

\section{Experimental}

\subsection{Synthesis of $3 D-P G$}

Graphite powders $(200 \mu \mathrm{m}$, 99.9\% purity) were purchased from Qingdao Dongkai Graphite Co., Ltd. All other chemicals were ordered from Tianjin Chemical Reagent Co., Ltd. and used directly without further purification. GO was prepared from graphite powder by a modified Hummers method[32]. 3D-PG was synthesized through a self-assembly hydrothermal method followed by annealing in nitrogen gas atmosphere. In a typical procedure, $50 \mathrm{~mL} \mathrm{GO}$ aqueous dispersion $\left(5 \mathrm{mg} \cdot \mathrm{mL}^{-1}\right)$ and 
$0.05 \mathrm{~mL}$ phytic acid (PA) solution $(60 \mathrm{wt} \%)$ were mixed under ultrasonication for 10 min to form a homogeneous suspension. The mixture was then transferred to a 100 $\mathrm{mL}$ Teflon-lined autoclave and heated at $180{ }^{\circ} \mathrm{C}$ for $12 \mathrm{~h}$ to generate a cylinderical hydrogel. After cooling down to room temperature, the hydrogel was washed with deionized water and freeze-dried overnight to remove the absorbed water of hydrogel. Finally, the obtained aerogel was annealed at $850{ }^{\circ} \mathrm{C}$ for $2 \mathrm{~h}$ in nitrogen gas atmosphere to afford the 3D-PG sample. For comparison, 3D graphene aerogel (3D-G) without P-doping was prepared through the same procedure without the addition of PA. 2D graphene (2D-G) was also prepared by directly annealing GO powders under the same annealing process.

\subsection{Electrochemical measurements and Instruments}

Cyclic voltammetric (CV), electrochemical impedance spectroscopy (EIS) and amperometric measurements were conducted on a PGSTAT-302N electrochemical workstation (Metrohm Ltd., Switzerland) using a conventional three-electrode system at room temperature. A modified glassy carbon electrode (GCE, $3 \mathrm{~mm}$ in diameter) served as the working electrode, $\mathrm{A} \mathrm{Ag} / \mathrm{AgCl}$ electrode and a $\mathrm{Pt}$ wire were used as reference and counter electrodes, respectively. The working electrodes were constructed by loading the same amount of eletrocatalysts on the surface of GCE, in which 3D-PG or 3D-G was cut into the disk-shaped sample (3 $\mathrm{mm}$ in diameter and 1 $\mathrm{mm}$ in height) and immobilized on GCE by a Nafion solution (0.5 wt.\%) to obtain 3D-PG/GCE or 3D-G/GCE electrode, whereas the 2D-G/GCE was prepared by a simple casting method described in our previous work[33]. Scanning electron 
microscopy (SEM) was performed on a JSM-7401F instrument (JEOL Ltd., Japan). Transmission electron microscopy (TEM), high-resolution transmission electron microscopy (HRTEM), and scanning transmission electron microscopy (STEM) elemental mapping were carried out on a JEM-2100 instrument (JEOL Ltd., Japan). XPS analysis was conducted on a PHI 5000C ESCA system (Perkin-Elmer Ltd., USA). Raman spectra were recorded on an Alpha 300R Raman spectroscope (WITEC Ltd., Germany). Nitrogen adsorption/desorption isotherms were performed on an ASAP 2020 instrument (Micromeritics Ltd., USA). Electrical conductivity of the samples was measured by a four-point probe method. The samples $(20 \mathrm{mg})$ were first compressed into pellets with thickness of $1 \mathrm{~mm}$. The voltage-current (V-I) plots were recorded on a RTS-9 device (ZYYB Ltd., China) with metal electrodes attached to the ends of pellets. The electrodes were painted with silver paste on both sides of the pellets to decrease the contact resistance.

\subsection{Detection of $\mathrm{H}_{2} \mathrm{O}_{2}$ in living cells.}

HeLa cells (human cervical cancer cells) obtained from The First Affiliated Hospital of Hebei North University were maintained in Dulbecco's Modified Eagle Medium (DMEM) culture medium containing 10\% Fetal Bovine Serum, $100 \mathrm{U} \cdot \mathrm{mL}^{-1}$ penicillin and $100 \mathrm{mg} \cdot \mathrm{mL}^{-1}$ streptomycin in an incubator at $37{ }^{\circ} \mathrm{C}$ with $5 \% \mathrm{CO}_{2}$ in a 95\% humidified atmosphere. After the growth of $\sim 90 \%$ confluence, the cells were separated from culture medium by centrifugation to obtain a cell-packed pellet, which was further washed with PBS ( $\mathrm{pH}$ 7.4) solution three times. The cell pellet resuspended in $20 \mathrm{~mL}$ of PBS $(0.1 \mathrm{M}, \mathrm{pH} 7.4)$ was then used as an electrolyte for the 
amperometric experiments. N-formylmethionyl-leucyl-pheny-lalanine (fMLP, 97\%) and catalase $\left(1000 \mathrm{U} \cdot \mathrm{mL}^{-1}\right)$ were used as $\mathrm{H}_{2} \mathrm{O}_{2}$ stimulating agent and scavenger, respectively.

\section{Results and discussion}

\subsection{Characterizations of $3 D-P G$}

The fabrication procedure of 3D-PG is illustrated in Fig. 1. In brief, GO is firstly mixed with PA in deionized water, where abundant oxygen-containing functional groups on GO tend to bind PA molecules via electrostatic attraction to form a homogeneous GO/PA suspension[34]. Subsequent hydrothermal process enables the partial GO reduction and self-assembly of reduced GO (RGO)/PA mixture to 3D-RGO/PA hydrogel (Fig. S1a, Supporting Information) driven by $\pi-\pi$ stacking, hydrogen bonding, electrostatic attraction and hydrophobic interactions[16]. The final high-temperature annealing leads to the simultaneous decomposition of PA into highly reactive $\mathrm{P}$ species, and complete removal of oxygen-containing groups of GO to generate defective/active sites on graphene[35]. As a result, $\mathrm{P}$ species are diffused and interacted with the defective sites of graphene lattices to achieve P-doping of 3D graphene, resulting in 3D-PG (Fig. S1b, Supporting Information). The as-prepared cylindrical 3D-PG is ultra-light with a bulk density of $3.8 \mathrm{mg} \cdot \mathrm{cm}^{-3}$, and can stand stably on the leaf of a chrysanthemum, without deforming it at all (Fig. S1c, Supporting Information). In addition, 3D-PG possesses a good compressive strength. As shown in Fig. S1d (Supporting Information), after compression, the cylindrical 3D-PG is able to completely recover to its original shape without collapsing. 
The morphology and structure of 3D-PG were examined by SEM/TEM, as shown in Fig. 2. SEM image (Fig. 2a) of 3D-PG shows a 3D architecture comprised of plentiful graphene sheets interconnected into a highly porous structure. Such 3D interconnected porous structures could effectively inhibit the restacking of graphene nanosheets and afford more pathways to facilitate direct transfer of electrons and electrolyte ions during the electrocatalysis[36]. High magnification of SEM (Fig. 2b) and TEM images (Fig. 2c) show that the 3D-PG is composed of the wrinkled and folded graphene sheets. These wrinkled/folded features might be caused by the removal of oxygen-containing groups on GO and heteroatoms doping [37]. A close examination from Fig. $2 \mathrm{c}$ reveals the presence of numerous mesopores $(\sim 5-10 \mathrm{~nm})$ in 3D-PG (marked by dotted circles). Selective area electron diffraction (SAED) image (Fig. 2c, inset) indicates the spot and ring patterns likely due to the deformation of the carbon structure induced by heteroatom doping [24]. HRTEM image (Fig. 2d) shows that the 3D-PG consists of few-layered (< 5 layers) graphene.

The doping structures of 3D-PG were investigated by XPS and Raman. A XPS survey scan of 3D-PG reveals four characteristic peaks of C1s, O1s, and P2p/P2s (Fig. 3a), while $\mathrm{P} 2 \mathrm{p} / \mathrm{P} 2 \mathrm{~s}$ peaks are absent for undoped $3 \mathrm{D}-\mathrm{G}$ and $2 \mathrm{D}-\mathrm{G}$ samples. In addition, the high-resolution P2p peak can be divided into two characteristic peaks at $131.7 \mathrm{eV}$ and $133.8 \mathrm{eV}$, arising from the $\mathrm{P}-\mathrm{C}$ and $\mathrm{P}-\mathrm{O}$ bonds [25], respectively (Fig. 3b). The large portion of $\mathrm{P}-\mathrm{C}$ bond indicates the incorporated $\mathrm{P}$ atoms are mainly bonded with $\mathrm{C}$ atoms at the defective sites of graphene. Furthermore, STEM elemental mapping (Fig. 3c) shows homogeneous distributions of $\mathrm{C}, \mathrm{O}$ and $\mathrm{P}$ elements throughout the 
selected region of 3D-PG. These results confirm that $\mathrm{P}$ atoms are successfully doped into the graphitic structures of 3D-PG. The carbon structures of 3D-PG were further studied by Raman spectroscopy, as shown in Fig. 3d. The Raman spectra show $G$-band at $\sim 1590 \mathrm{~cm}^{-1}$ and $D$-band at $\sim 1350 \mathrm{~cm}^{-1}$. The $G$-band is assigned to graphitic carbons and the $D$-band corresponds to the disorder or defects present in the graphitic domains $[38,39]$. The $I(D) / I(G)$ intensity ratio is a qualitative measure of the defect density in carbon materials, and the $I(D) / I(G)$ of 3D-PG (1.18) is apparently higher than that of 3D-G (1.05) and 2D-G (1.01), suggesting that more defects are introduced in 3D-PG attributed to the incorporation of $\mathrm{P}$ atoms. To further confirm the defect type of 3D-PG, the full-width at half-maximum of $\mathrm{G}$ band $\left(G_{\mathrm{FWHM}}\right)$ are determined, yielding $33.6,34.2$ and $34.8 \mathrm{~cm}^{-1}$ for 2D-G, 3D-G and 3D-PG, respectively, indicating that both 3D-assambly and P-doping have little impact on $G_{\text {FWHM. }}$. It is well documented that the $I(D) / I(G)$ correlates well with the carbon crystalline size (inverse proportional relationship) while $G_{\text {FWHM }}$ is highly dependent on the amount of $s p^{3}$ amorphous carbon (proportional relationship)[40]. The increased $I(D) / I(G)$ and negligibly changed $G_{\text {FWHM }}$ of 3D-G and 3D-PG with respect to 2D-G demonstrate that the defects derived from 3D-assambly and P-doping are associated with the reduced carbon crystalline size rather than the generation of $s p^{3}$ amorphous carbon. Moreover, the $G$ band position of 2D-G $\left(1607 \mathrm{~cm}^{-1}\right)$ remains almost unchanged after 3D-assambly $\left(1610 \mathrm{~cm}^{-1}\right)$ but down-shifts to $1596 \mathrm{~cm}^{-1}$ after P-doping, which is attributed to phonon-stiffening induced by P-doping[41], consistent with other reports for heteroatom-doped graphene materials[41-43]. 
Nitrogen adsorption/desorption analysis was employed to examine the porous structures and specific surface area of 3D-PG. As shown in Fig. 4a, a typical characteristic of type-IV isotherms with a distinct hysteresis loop implies the presence of hierarchical macro/meso-pores in the frameworks of 3D-PG. This is further confirmed by the corresponding pore size distribution obtained from a Barrett-Joyner-Halenda (BJH, [44]) model (Fig. 4a, inset), showing that much of the pore volume lies in the range of $2-70 \mathrm{~nm}$ with a peak mesopore diameter of $8.2 \mathrm{~nm}$, consistent with the TEM observations (Fig. 2c). In addition, 3D-PG possesses a Brunauer-Emmett-Teller (BET, [45]) specific surface area of $686.2 \mathrm{~m}^{2} \cdot \mathrm{g}^{-1}$, much higher than that of $2 \mathrm{D}-\mathrm{G}\left(287.6 \mathrm{~m}^{2} \cdot \mathrm{g}^{-1}\right.$, Table S1, Supporting Information) attributed mainly to the unique 3D porous architecture that can effectively prevent the restacking of graphene sheets. Note that the surface area of 3D-PG is also slightly higher than that of 3D-G $\left(581.6 \mathrm{~m}^{2} \cdot \mathrm{g}^{-1}\right.$, Table S1, Supporting Information). This indicates that the P-doping is helpful for the enlarged BET surface area, which can be explained by the decomposition of PA precursors during the annealing process that can act in a porogen manner for generating more macro/meso-pores[28]. The high surface area of 3D-G together with its hierarchical macro/meso-pores structure is favorable for the eletrocatalytic performance as it can provide a large accessible area and efficient electron/ion-transport channels.

Fig. $4 \mathrm{~b}$ compares the $I-V$ curves of the samples and the electrical conductivity values of the samples are listed in Table S1 (Supporting Information). It is obvious that the conductivity of 3D-PG $\left(2.17 \mathrm{~S} \cdot \mathrm{cm}^{-1}\right)$ or $3 \mathrm{D}-\mathrm{G}\left(1.53 \mathrm{~S} \cdot \mathrm{cm}^{-1}\right)$ is nearly 10 
times larger than that of $2 \mathrm{D}-\mathrm{G}\left(0.18 \mathrm{~S} \cdot \mathrm{cm}^{-1}\right)$. This is attributed the $3 \mathrm{D}$ well-interconnected percolated graphene networks that can greatly reduce the inter-sheet resistance between graphene sheets[15]. More interestingly, the conductivity of 3D-PG $\left(2.17 \mathrm{~S} \cdot \mathrm{cm}^{-1}\right)$ is slightly higher than that of 3D-G $(1.53$ $\mathrm{S} \cdot \mathrm{cm}^{-1}$ ), demonstrating the positive role of P-doping in enhancing the conductivity. Similar phenomena can also be observed in other reported heteroatom-doped materials [30, 46, 47]. It has been reported that P-doping is able to improve the electron-donor properties and increase the density of conducting electrons of carbon at energies near Fermi level[30], consequently enhancing the electron transfer capability and conductivity of 3D-PG.

\subsection{Application of 3D-PG for $\mathrm{H}_{2} \mathrm{O}_{2}$ sensing}

$\mathrm{H}_{2} \mathrm{O}_{2}$ is a powerful oxidizing agent and an essential intermediate that is widely applied in environmental, pharmaceutical, biomedical, clinical, and many other fields [48], and thus the reliable, rapid and accurate detection of the trace amount of $\mathrm{H}_{2} \mathrm{O}_{2}$ is of great importance. The catalytic activity of electrocatalyst for $\mathrm{H}_{2} \mathrm{O}_{2}$ reduction reaction (HRR) plays a significant role in determining the $\mathrm{H}_{2} \mathrm{O}_{2}$ sensing performance, and many studies report graphene-based materials are promising HRR electrocatalysts [4]. Thanks to the unique 3D porous structure combined with P-doping, 3D-PG is believed to exhibit an enhanced HRR performance and sensitive detection of $\mathrm{H}_{2} \mathrm{O}_{2}$.

Fig. 5a displays the CVs of 3D-PG/GCE, 3D-G/GCE and 2D-G/GCE electrodes $\underline{\text { in }}_{2}$-saturated PBS (0.1 M, pH 7.4) with the addition of $1.0 \mathrm{mM} \mathrm{H}_{2} \underline{\mathrm{O}}_{2}$. As seen, all $\underline{\text { the electrodes show well-defined reduction peaks at potentials of }-0.2 \sim-0.3 \mathrm{~V} \text { in the }}$ 
presence of $\mathrm{H}_{2} \underline{\mathrm{O}}_{2}$, suggesting their HRR activity. However, the 2D-G/GCE shows a weak HRR activity, as reflecting by its low peak current density at potential of -0.294 $\underline{\text { V. In contrast, the 3D-G/GCE exhibits a dramatically enhanced HRR activity with }}$ higher peak current density at more positive potential of $-0.268 \mathrm{~V}$. Obviously, the 3D-PG/GCE exhibits a highest HRR activity as evidenced by the most positive potential $(-0.233 \mathrm{~V})$ and largest peak current density as compared to those of 3D-G/GCE and 2D-G/GCE. The outstanding HRR activity of 3D-PG/GCE electrode can be attributed to the synergistic effects of 3D interconnected porous structures and P-doping for the following aspects. Firstly, benefiting from the 3D graphene networks and hierarchical macro/mesoporosity, 3D-PG has a large BET specific surface area (Fig. 4a \& Table S1, Supporting Information). Generally, the large BET specific $\underline{\text { surface area can provide a high electrochemically active surface area (ECSA) }}$ available for electrocatalytic reaction[49]. To confirmed this, the ECSA values are estimated by plotting the peak potentials $\left(I_{p}\right)$ with the square root of the scan rate $(v)$ derived from the $\mathrm{CVs}$ of all the electrodes in $0.1 \mathrm{M} \mathrm{KCl}$ solution containing $5.0 \mathrm{mM}$ $\left.\left[\mathrm{Fe}(\mathrm{CN})_{6}\right]^{3-}\right|^{4-}$ according to the Randled-Sevcik equation [50], as shown in Fig. S2 (Supporting Information). The ECSA values of the 3D-PG/GCE, 3D-G/GCE, and 2D-G/GCE electrodes are calculated to be $0.59,0.25$, and $0.09 \mathrm{~cm}^{2}$, respectively. The highest ECSA value suggests that the 3D-PG exposes the largest number of effective $\underline{\text { active sites, which facilitate the high efficiency in } \mathrm{H}_{2} \underline{\mathrm{O}}_{2}}$ adsorption and reduction, leading to the excellent HER activity of 3D-PG/GCE. Secondly, the synergistic effects between 3D interconnected structure and P-doping greatly enhance the electric 
conductivity, as verified in Fig. $4 \mathrm{~b} \&$ Table S1. This can also be observed from the EIS analysis (Fig. S3, Supporting Information), which shows the charge transfer resistance decreases significantly for 3D-PG/GCE electrode as compared to 3D-G/GCE and 2D-G/GCE electrodes. The excellent electrical conductivity of the electrode enables a rapid electron and ion transport at the catalyst-electrolyte interfaces, facilitating the HRR electrocatalytic kinetics. Thirdly, Raman analysis (Fig. 3d) reveals that the P-doping induces more disorders of the carbon structure corresponding to a high level of defects on graphene. These defective sites facilitate effective HRR activities as they can directly act as active sites for electrocatalysis. Hence the P-doping provides additional electroactive sites that contribute to a better electrochemical performance. Therefore, the synergistic effects of 3D porous structures and P-doping result in enlarged ECSA, improved charge transfer, and generation of abundant defective/active sites, leading to the significantly enhanced HRR activity.

Because of the outstanding electrocatalytic activity for HRR, the developed 3D-PG/GCE was then employed as an electrode material for $\mathrm{H}_{2} \mathrm{O}_{2}$ sensing. In order to ensure an optimal amperometric response of an electrochemical sensor, the applied potentials for amperometric detection of $\mathrm{H}_{2} \mathrm{O}_{2}$ is optimized as $-0.4 \mathrm{~V}$ (Fig. S4, Supporting Information). Fig. 5b shows the typical steady-state amperometric response of the 3D-PG/GCE electrode to the successive addition of $\mathrm{H}_{2} \mathrm{O}_{2}$ in stirring $\mathrm{N}_{2}$-saturated PBS (0.1 M, pH 7.4) at $-0.4 \mathrm{~V}$. It is obvious that the addition of $\mathrm{H}_{2} \mathrm{O}_{2}$ results in a well-defined stepwise increment in the current responses. As seen in the 
inset of Fig. 5b, the steady-state current reaches $95 \%$ of the maximum is less than $2 \mathrm{~s}$ and the discernable response to the $\mathrm{H}_{2} \mathrm{O}_{2}$ concentration can be as low as $0.2 \mu \mathrm{M}$, demonstrating the rapid and highly sensitive current response of the 3D-PG/GCE sensor. As shown in Fig. 5c, the calibration curve of the current versus $\mathrm{H}_{2} \mathrm{O}_{2}$ concentration indicates that the sensing signal increases linearly with $\mathrm{H}_{2} \mathrm{O}_{2}$ concentration in the range of $0.2 \mu \mathrm{M}$ to $41.2 \mathrm{mM}$. From the slope of the calibration curve, the limit of detection (LOD) is estimated to be $0.17 \mu \mathrm{M}(\mathrm{S} / \mathrm{N}=3)$, consistent with the amperometric detection of trace-level $\mathrm{H}_{2} \mathrm{O}_{2}$ (Fig. 5b, inset). Such sensing performances of the 3D-PG/GCE sensor compare favorably to those of most reported graphene-based $\mathrm{H}_{2} \mathrm{O}_{2}$ sensors (Table $\mathrm{S} 1$, Supporting Information). It is suggested that the strong HRR activity of 3D-PG due to the synergistic effects of $3 \mathrm{D}$ porous structures and P-doping contributes to the good $\mathrm{H}_{2} \mathrm{O}_{2}$ sensing performances of 3D-PG/GCE.

High selectivity is critically important in view of the practical use of 3D-PG/GCE. The common foreign interfering species such as glucose, uric acid (UA), dopamine (DA), and ascorbic acid (AA) are chosen to evaluate the selectivity of 3D-PG/GCE. As shown in Fig. 5d, the successive addition of interferences including $1.0 \mathrm{mM}$ glucose, $1.0 \mathrm{mM}$ UA, $1.0 \mathrm{mM}$ DA, and $1.0 \mathrm{mM}$ AA do not induce an obvious current change, while a sharp decrease in current upon the addition of $1.0 \mathrm{mM} \mathrm{H}_{2} \mathrm{O}_{2}$, suggesting that the 3D-PG/GCE exhibits a good selectivity for $\mathrm{H}_{2} \mathrm{O}_{2}$ detection. The reproducibility and stability of 3D-PG/GCE were also evaluated. The reproducibility of the 3D-PG/GCE was studied by comparing the current response of five electrodes 
prepared under the same conditions, and the relative standard deviation (RSD) is $1.72 \%$, implying a good reproducibility. To investigate the long-term stability of the sensor, the 3D-PG/GCE was stored at $4{ }^{\circ} \mathrm{C}$ in refrigerator and the current response to $1.0 \mathrm{mM} \mathrm{H}_{2} \mathrm{O}_{2}$ was determined every two day. After ten periods (20 days), the current response still retains about $93.1 \%$ of its initial response (Fig. S5, Supporting Information), indicating that the sensor is very stable. These results suggest that the present 3D-PG/GCE sensor can be used for sensitive, selective and stable detection of $\mathrm{H}_{2} \mathrm{O}_{2}$.

Owing to the excellent electrochemical sensing performances of 3D-PG/GCE, 3D-PG/GCE was then used in biological systems for real-time detection of trace-level $\mathrm{H}_{2} \mathrm{O}_{2}$ in living HeLa cells. fMLP was employed as a stimulating agent to induce the release of $\mathrm{H}_{2} \mathrm{O}_{2}$ from living $\mathrm{HeLa}$ cells. The experiments were performed by the same amperometric technique in $\mathrm{N}_{2}$-saturated PBS $(0.1 \mathrm{M}, \mathrm{pH} 7.4)$ at the applied potential of $-0.4 \mathrm{~V}$. As shown in Fig. 6, a sharp increase in the current is observed for the 3D-PG/GCE electrode upon the addition of $10 \mathrm{mM}$ fMLP in living HeLa cells, which further decreases progressively to the level of background signal after the addition of $100 \mathrm{U} \cdot \mathrm{mL}^{-1}$ catalase known as $\mathrm{H}_{2} \mathrm{O}_{2}$ scavenger. In contrast, for control wells containing no HeLa cells, the amperometric response remains almost unchanged after sequential addition of the same amount of fMLP and catalase. These results indicate that the increased amperometric response is attributed only to the $\mathrm{H}_{2} \mathrm{O}_{2}$ released from the HeLa cells that stimulated by fMLP. Furthermore, the amount of released $\mathrm{H}_{2} \mathrm{O}_{2}$ released from HeLa cells can be determined as $0.87 \mu \mathrm{M}$ by measuring the maximum 
current change based on the calibration curve as obtained in Fig. 5c. This demonstrates that the 3D-PG/GCE sensor can be practically used for the sensitive detection of $\mathrm{H}_{2} \mathrm{O}_{2}$ in living cells.

\section{Conclusions}

In summary, 3D-PG was successfully synthesized by a combined self-assembly hydrothermal method and subsequent annealing process, as characterized by SEM/TEM, XPS, Raman, BET and conductivity measurements. Electrochemical measurements showed that 3D-PG/GCE presented a significantly improved HRR activity as compared to 3D-G/GCE and 2D-G/GCE, which was attributed to the synergistic effects of $3 \mathrm{D}$ porous framework and P-doping that resulted in enlarged $\underline{\text { ECSA}}$, enhanced charge transfer, and generation of abundant defective/active sites. As a result, the 3D-G/GCE based $\mathrm{H}_{2} \mathrm{O}_{2}$ sensor exhibited a wide linear range of $0.0002-41.2 \mathrm{mM}$ and a low detection limit of $0.17 \mu \mathrm{M}$, together with the excellent performances in selectivity, reproducibility, and long-term stability. Moreover, the 3D-G/GCE sensor could be practically used to accurately determine a trace amount of $\mathrm{H}_{2} \mathrm{O}_{2}$ released from living $\mathrm{HeLa}$ cells. These results highlight the significant structure/chemical design of 3D graphene with heteroatoms doping for developing high-performance electrochemical sensors.

\section{Acknowledgement}

This work is supported by "Engineering Technology Research Center of Population Health Informatization in Hebei Province", "Application Technology Research and Development Center of Medical Informatics in Hebei Universities", 
"Institute of New Energy Science and Technology of Hebei North University", Science and Technology Department of Hebei Province Projects (No.16961301D and No.152111230), Health Department of Hebei Province Project (No.20160030) and National Science Foundation of China (No.51501083).

\section{References}

[1] E. Bakker, Electrochemical sensors, Anal. Chem., 76 (2004) 3285-3298.

[2] Y. Shao, J. Wang, H. Wu, J. Liu, I.A. Aksay, Y. Lin, Graphene based electrochemical sensors and biosensors: a review, Electroanalysis, 22 (2010) 1027-1036.

[3] J. Wang, Electrochemical biosensing based on noble metal nanoparticles, Microchim. Acta, 177 (2012) 245-270.

[4] X. Luo, A. Morrin, A.J. Killard, M.R. Smyth, Application of nanoparticles in electrochemical sensors and biosensors, Electroanalysis, 18 (2006) 319-326.

[5] A.T. Lawal, Synthesis and utilisation of graphene for fabrication of electrochemical sensors, Talanta, 131 (2015) 424-443.

[6] S. Liu, J. Tian, L. Wang, H. Li, Y. Zhang, X. Sun, Stable aqueous dispersion of graphene nanosheets: noncovalent functionalization by a polymeric reducing agent and their subsequent decoration with $\mathrm{Ag}$ nanoparticles for enzymeless hydrogen peroxide detection, Macromolecules, 43 (2010) 10078-10083.

[7] Y. Song, K. Qu, C. Zhao, J. Ren, X. Qu, Graphene oxide: intrinsic peroxidase catalytic activity and its application to glucose detection, Adv. Mater., 22 (2010) 2206-2210.

[8] Y. Wang, Y. Li, L. Tang, J. Lu, J. Li, Application of graphene-modified electrode for selective detection of dopamine, Electrochem. Commun., 11 (2009) 889-892.

[9] X. Kang, J. Wang, H. Wu, J. Liu, I.A. Aksay, Y. Lin, A graphene-based electrochemical sensor for sensitive detection of paracetamol, Talanta, 81 (2010) 754-759.

[10] C.X. Guo, Z.S. Lu, Y. Lei, C.M. Li, Ionic liquid-graphene composite for ultratrace explosive trinitrotoluene detection, Electrochem. Commun., 12 (2010) 1237-1240.

[11] F. Liu, Y. Piao, J.S. Choi, T.S. Seo, Three-dimensional graphene micropillar based electrochemical sensor for phenol detection, Biosens. Bioelectron., 50 (2013) 387-392.

[12] C. Li, G. Shi, Three-dimensional graphene architectures, Nanoscale, 4 (2012) 5549-5563.

[13] Z. Chen, W. Ren, L. Gao, B. Liu, S. Pei, H.M. Cheng, Three-dimensional flexible and conductive interconnected graphene networks grown by chemical vapour deposition, Nature Mater., 10 (2011) 424-428.

[14] X. Cao, Z. Yin, H. Zhang, Three-dimensional graphene materials: preparation, structures and application in supercapacitors, Energ. Environ. Sci., 7 (2014) 1850-1865.

[15] X. Dong, Y. Cao, J. Wang, M.B. Chan-Park, L. Wang, W. Huang, P. Chen, Hybrid structure of zinc oxide nanorods and three dimensional graphene foam for supercapacitor and electrochemical sensor applications, RSC Adv., 2 (2012) 4364-4369.

[16] H. Liu, Y. Liu, D. Zhu, Chemical doping of graphene, J. Mater. Chem., 21 (2011) 3335-3345.

[17] L.T. Qu, Y. Liu, J.B. Baek, L.M. Dai, Nitrogen-doped graphene as efficient metal-free electrocatalyst for oxygen reduction in fuel cells, ACS Nano, 4 (2010) 1321-1326. 
[18] X.W. Wang, G.Z. Sun, P. Routh, D.H. Kim, W. Huang, P. Chen, Heteroatom-doped graphene materials: syntheses, properties and applications, Chem. Soc. Rev., 43 (2014) 7067-7098.

[19] Z.X. Cai, X.H. Song, Y.Y. Chen, Y.R. Wang, X. Chen, 3D nitrogen-doped graphene aerogel: A low-cost, facile prepared direct electrode for $\mathrm{H}_{2} \mathrm{O}_{2}$ sensing, Sens. Actual. B: Chem, 222 (2016) 567-573.

[20] J. Guo, T. Zhang, C. Hu, L. Fu, A three-dimensional nitrogen-doped graphene structure: a highly efficient carrier of enzymes for biosensors, Nanoscale, 7 (2015) 1290-1295.

[21] X. Feng, Y. Zhang, J. Zhou, Y. Li, S. Chen, L. Zhang, Y. Ma, L. Wang, X. Yan, Three-dimensional nitrogen-doped graphene as an ultrasensitive electrochemical sensor for the detection of dopamine, Nanoscale, 7 (2015) 2427-2432.

[22] H. Wang, T. Maiyalagan, X. Wang, Review on recent progress in nitrogen-doped graphene: synthesis, characterization, and its potential applications, ACS Catal., 2 (2012) 781-794.

[23] C. Zhang, N. Mahmood, H. Yin, F. Liu, Y. Hou, Synthesis of phosphorus-doped graphene and its multifunctional applications for oxygen reduction reaction and lithium ion batteries, Adv. Mater., 25 (2013) 4932-4937.

[24] R. Li, Z. Wei, X. Gou, W. Xu, Phosphorus-doped graphene nanosheets as efficient metal-free oxygen reduction electrocatalysts, RSC Adv., 3 (2013) 9978-9984.

[25] P. Karthika, N. Rajalakshmi, K. Dhathathreyan, Phosphorus-doped exfoliated graphene for supercapacitor electrodes, J. Nanosci. Nanotechno., 13 (2013) 1746-1751.

[26] F. Razmjooei, K.P. Singh, E.J. Bae, J.S. Yu, A new class of electroactive Fe-and P-functionalized graphene for oxygen reduction, J. Mater. Chem. A, 3 (2015) 11031-11039.

[27] Y. Wen, B. Wang, C. Huang, L. Wang, D. Hulicova-Jurcakova, Synthesis of phosphorus-doped graphene and its wide potential window in aqueous supercapacitors, Chem. Eur. J., 21 (2015) 80-85.

[28] X. Ma, G. Ning, C. Qi, C. Xu, J. Gao, Phosphorus and nitrogen dual-doped few-layered porous graphene: a high-performance anode material for lithium-ion batteries, ACS Appl. Mater. Inter., 6 (2014) 14415-14422.

[29] W. Chen, L. Yan, In situ self-assembly of mild chemical reduction graphene for three-dimensional architectures, Nanoscale, 3 (2011) 3132-3137.

[30] D. Li, M.B. Müller, S. Gilje, R.B. Kaner, G.G. Wallace, Processable aqueous dispersions of graphene nanosheets, Nature Nanotechnol., 3 (2008) 101-105.

[31] Y. Tian, Y. Ma, H. Liu, X. Zhang, W. Peng, One-step and rapid synthesis of nitrogen and sulfur co-doped graphene for hydrogen peroxide and glucose sensing, J. Electroanal. Chem., 742 (2015) 8-14.

[32] D. Chen, H. Feng, J. Li, Graphene oxide: preparation, functionalization, and electrochemical applications, Chem. Rev., 112 (2012) 6027-6053.

[33] X. Li, H. Wang, J.T. Robinson, H. Sanchez, G. Diankov, H. Dai, Simultaneous nitrogen doping and reduction of graphene oxide, J. Am. Chem. Soc., 131 (2009) 15939-15944.

[34] J. Liang, Y. Jiao, M. Jaroniec, S.Z. Qiao, Sulfur and nitrogen dual-doped mesoporous graphene electrocatalyst for oxygen reduction with synergistically enhanced performance, Angew. Chem. Int. Edit., 51 (2012) 11496-11500.

[35] A.G. Kannan, J.X. Zhao, S.G. Jo, Y.S. Kang, D.W. Kim, Nitrogen and sulfur co-doped graphene counter electrodes with synergistically enhanced performance for dye-sensitized solar cells, J. Mater. Chem. A, 2 (2014) 12232-12239.

[36] A. Ferrari, J. Meyer, V. Scardaci, C. Casiraghi, M. Lazzeri, F. Mauri, S. Piscanec, D. Jiang, K. Novoselov, S. Roth, Raman spectrum of graphene and graphene layers, Phys. Rev. Lett., 97 (2006) 
187401.

[37] E.P. Barrett, L.G. Joyner, P.P. Halenda, The determination of pore volume and area distributions in porous substances. I. Computations from nitrogen isotherms, J. Am. Chem. Soc., 73 (1951) 373-380.

[38] S. Brunauer, P.H. Emmett, E. Teller, Adsorption of gases in multimolecular layers, J. Am. Chem. Soc., 60 (1938) 309-319.

[39] W. Ai, Z. Luo, J. Jiang, J. Zhu, Z. Du, Z. Fan, L. Xie, H. Zhang, W. Huang, T. Yu, Nitrogen and sulfur codoped graphene: multifunctional electrode materials for high-performance Li-ion batteries and oxygen reduction reaction, Adv. Mater., 26 (2014) 6186-6192.

[40] F. Razmjooei, K.P. Singh, M.Y. Song, J.S. Yu, Enhanced electrocatalytic activity due to additional phosphorous doping in nitrogen and sulfur-doped graphene: A comprehensive study, Carbon, 78 (2014) 257-267.

[41] A.A. Karyakin, E.E. Karyakina, L. Gorton, Amperometric biosensor for glutamate using Prussian blue-based "artificial peroxidase" as a transducer for hydrogen peroxide, Anal. Chem., 72 (2000) 1720-1723.

[42] K. Mayrhofer, D. Strmcnik, B.B. Blizanac, V. Stamenkovic, M. Arenz, N.M. Markovic, Measurement of oxygen reduction activities via the rotating disc electrode method: From Pt model surfaces to carbon-supported high surface area catalysts, Electrochim. Acta, 53 (2008) 3181-3188.

[43] K.B. Oldham, Analytical expressions for the reversible Randles-Sevcik function, J. Electroanal. Chem. Inter. Electrochem., 105 (1979) 373-375.

[44] C. Wu, S. Deng, H. Wang, Y. Sun, J. Liu, H. Yan, Preparation of novel three-dimensional $\mathrm{NiO} /$ ultrathin derived graphene hybrid for supercapacitor applications, ACS Appl. Mater. Inter., 6 (2014) 1106-1112. 


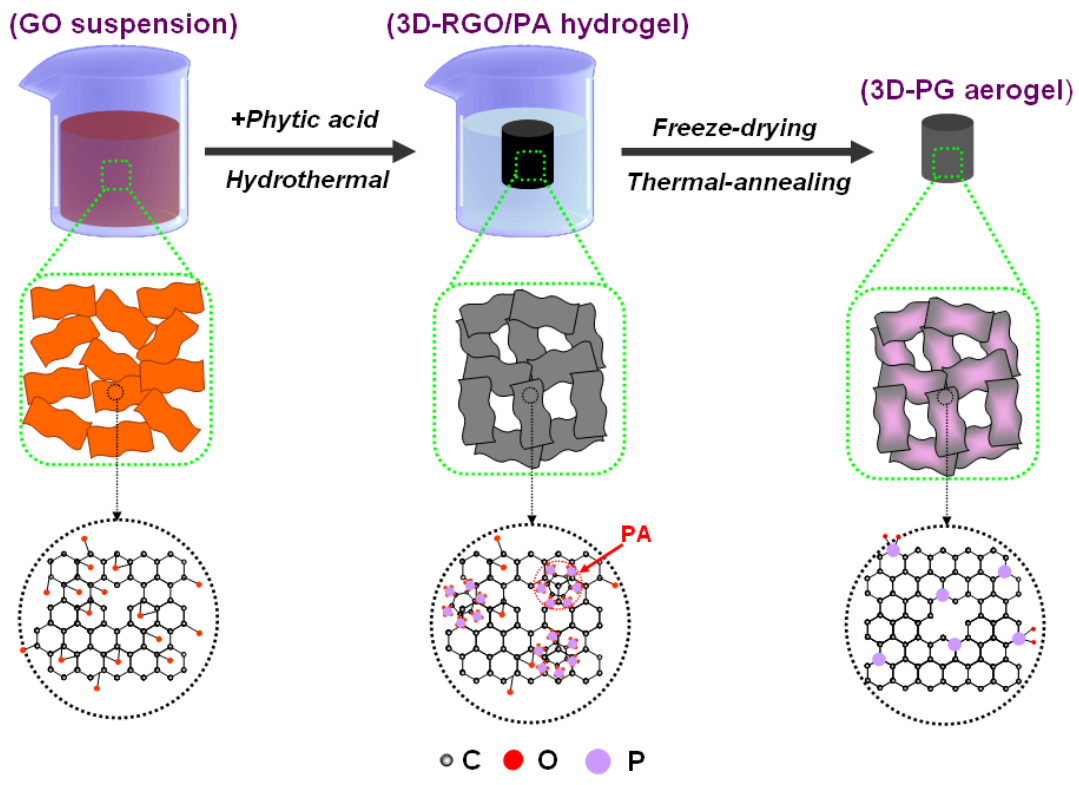

Fig. 1. Schematic illustration of the synthesis process of 3D-PG 

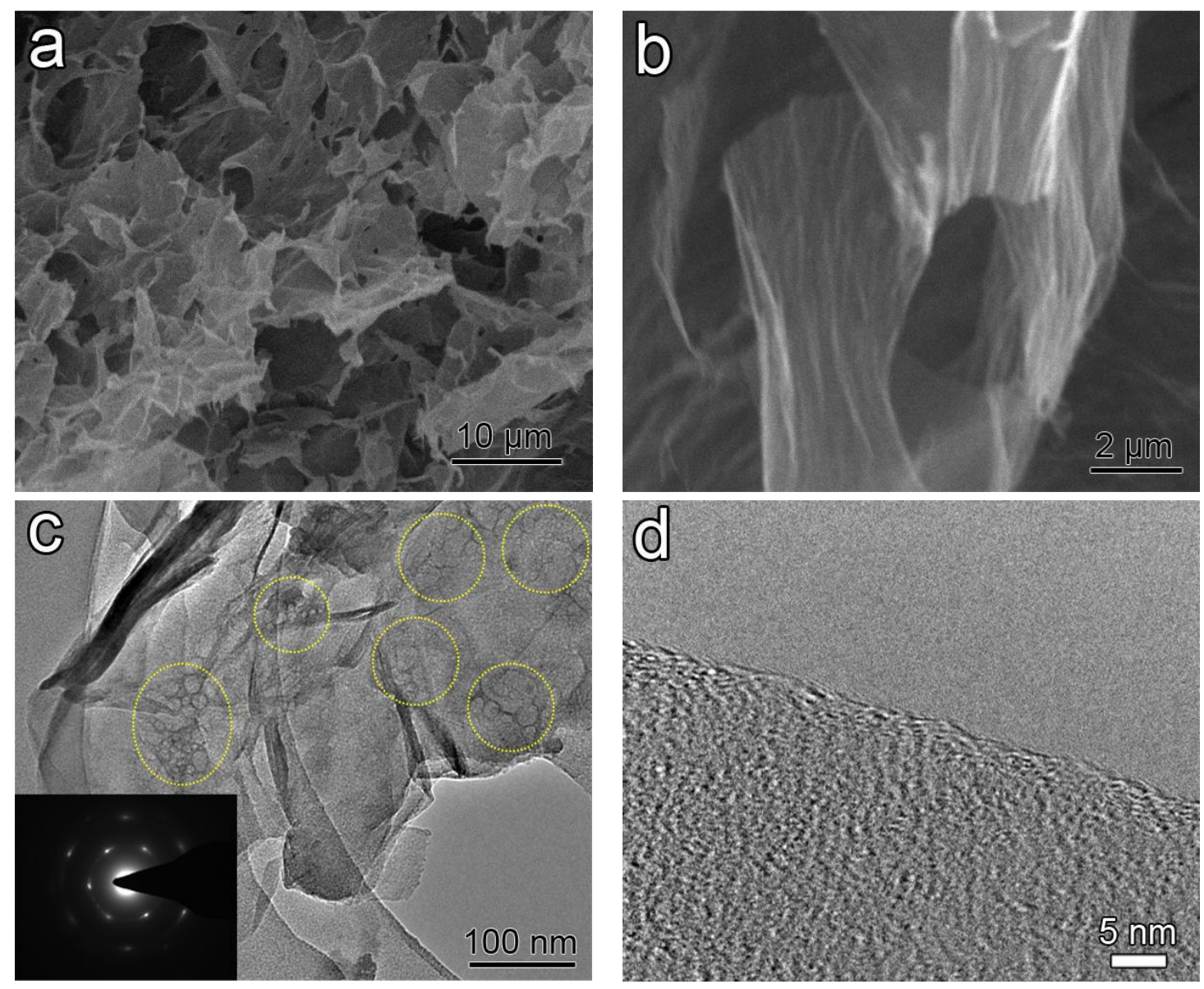

Fig. 2. Low (a) and high (b) magnification SEM image of as-prepared 3D-PG. (c) TEM image of 3D-PG (inset: corresponding SAED pattern). (d) HRTEM image of 3D-PG. 

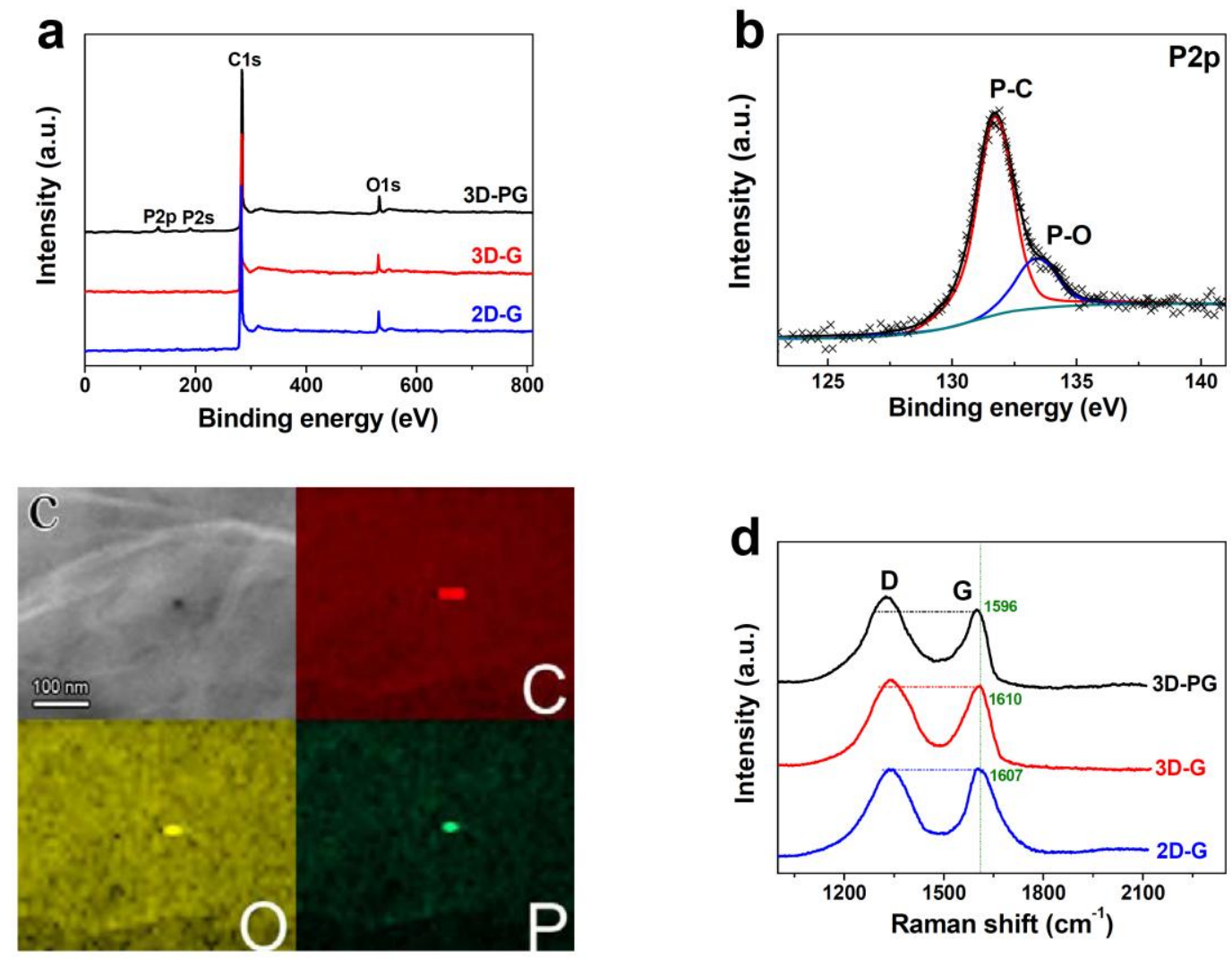

Fig. 3. (a) XPS survey scan of the 2D-G, 3D-G and 3D-PG samples. (b) High-resolution P2p spectrum of 3D-PG. (c) STEM image of 3D-PG and the corresponding elemental mapping images of C, O, and P. (d) Raman spectra of the 2D-G, 3D-G and 3D-PG samples. 

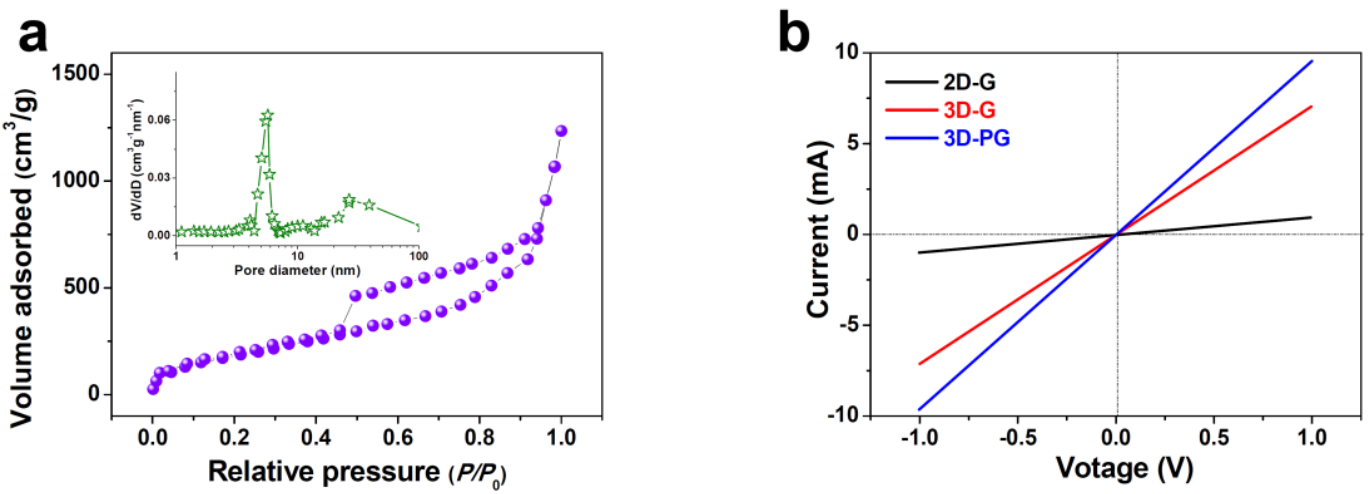

Fig. 4. (a) Nitrogen adsorption/desorption isotherm curve of 3D-PG (inset is the pore size distribution). (b) Current-voltage curves of 2D-G, 3D-G and 3D-PG 

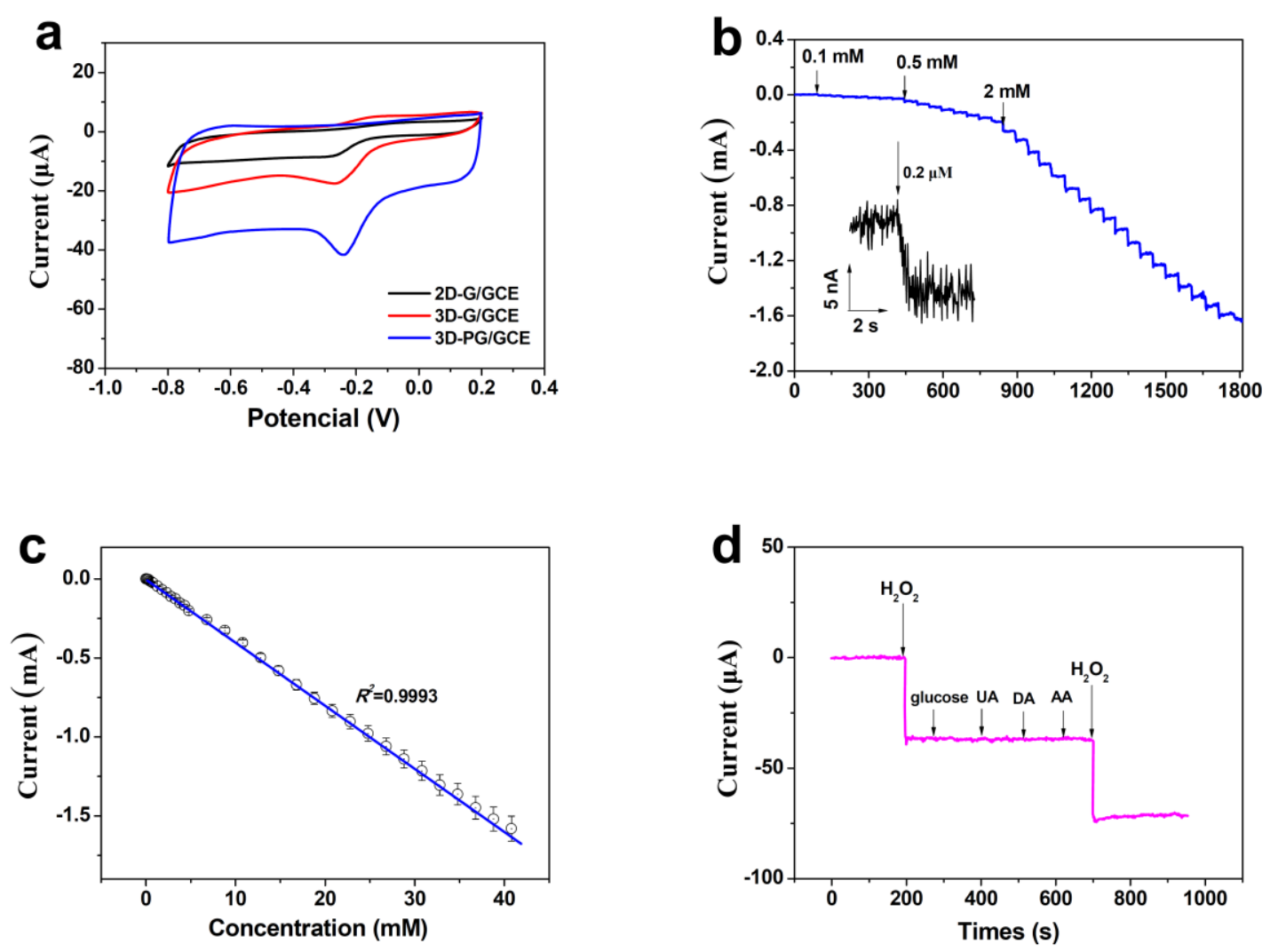

Fig. 5. (a) CVs of 2D-G/GCE, 3D-G/GCE and 3D-PG/GCE electrodes in $\mathrm{N}_{2}$-saturated PBS (0.1 M, pH 7.4) with addition of $1.0 \mathrm{mM} \mathrm{H}_{2} \mathrm{O}_{2}$ at the scan rate of $50 \mathrm{mV} / \mathrm{s}$. (b) Amperometric response of 3D-PG/GCE sensor to successive addition of $\mathrm{H}_{2} \mathrm{O}_{2}$ to $\mathrm{N}_{2}$-saturated PBS $(0.1 \mathrm{M}, \mathrm{pH} 7.4)$ at $-0.4 \mathrm{~V}$. Inset: amperometric response to $0.2 \mu \mathrm{M}$ $\mathrm{H}_{2} \mathrm{O}_{2}$. (c) Calibration plot of current versus $\mathrm{H}_{2} \mathrm{O}_{2}$ concentration. (d) Interference test of the 3D-PG/GCE sensor to the successive addition of $1.0 \mathrm{mM} \mathrm{H}_{2} \mathrm{O}_{2}, 1.0 \mathrm{mM}$ glucose, $1.0 \mathrm{mM}$ UA, $1.0 \mathrm{mM}$ DA, $1.0 \mathrm{mM}$ AA and $1.0 \mathrm{mM} \mathrm{H}_{2} \mathrm{O}_{2}$. 


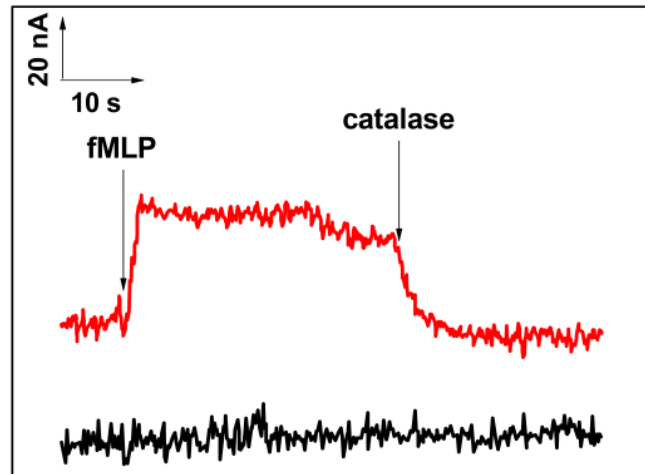

Fig. 6. Amperometric response of the 3D-PG/GCE electrode upon sequential addition of $10 \mathrm{mM}$ fMLP and $100 \mathrm{U} \cdot \mathrm{mL}^{-1}$ catalase to $\mathrm{N}_{2}$-saturated PBS $(0.1 \mathrm{M}, \mathrm{pH} 7.4)$ at -0.4 $\mathrm{V}$ in the presence (upper) and absence (lower) of HeLa cells. 


\section{Author Biographies}

Ye Tian is an associate professor at Hebei North University. Her current interests are graphene-based materials and their electrocatalysis applications.

Wei Zhen is an associate professor at Hebei North University. Her main research fields are organic synthesis and electrochemical sensors.

Kehui Zhang is an experimentalist at Hebei University of Architecture and Engineering. His research includes nanomaterials and their electrocatalysis applications.

Shuo Peng is a professor at Hebei North University. His current interests are graphene-based materials and their electrocatalysis applications.

Xiao Zhang is a professor at Hebei North University. His current interests are graphene-based electrochemical sensors.

Wuming Liu is a professor at Institute of Physics, Chinese Academy of Sciences. His research includes condensed matter theory and theoretical study on the electronic structure of graphene.

Ke Chu is an associate professor at Lanzhou Jiaotong University. His current interests are graphene-based materials and their applications in nanocomposites and electrocatalysis. 